\title{
Heterogenous Catalysis Mediated by Plasmon Heating
}

\author{
James R. Adleman, ${ }^{\dagger}$ David A. Boyd, ${ }^{*, \dagger}$ David G. Goodwin,${ }^{\dagger}$ and Demetri Psaltis ${ }^{\dagger, \ddagger}$ \\ Division of Engineering and Applied Science, California Institute of Technology, \\ Pasadena, California 91125, and Ecole Polytechnique Fédérale de Lausanne, \\ Lausanne, Switzerland
}

Received August 19, 2009; Revised Manuscript Received October 16, 2009

\begin{abstract}
We introduce a new method for performing and miniaturizing many types of heterogeneous catalysis involving nanoparticles. The method makes use of the plasmon resonance present in nanoscale metal catalysts to provide the necessary heat of reaction when illuminated with a low-power laser. We demonstrate our approach by reforming a flowing, liquid mixture of ethanol and water over gold nanoparticle catalysts in a microfluidic channel. Plasmon heating of the nanoparticles provides not only the heat of reaction but the means to generate both water and ethanol vapor locally over the catalysts, which in turn allows the chip and the fluid lines to remain at room temperature. The measured products of the reaction, $\mathrm{CO}_{2}, \mathrm{CO}$, and $\mathrm{H}_{2}$, are consistent with catalytic steam reforming of ethanol. The approach, which we refer to as plasmon-assisted catalysis, is general and can be used with a variety of endothermic catalytic processes involving nanoparticles.
\end{abstract}

Heterogeneous catalysis is of critical importance in a variety of chemical, environmental, and energy-conversion processes. A central component in both research and industrial catalysis systems is the continuous flow reactor. Typically, this is a macroscopic vessel in which a continuous stream of reactants is converted to a desired product via contact with a catalyst. The reactors generally run under open-loop control, and the conditions, e.g., temperature and mass flow rates, are optimized to generate reaction products at welldefined rates. Conversely, one can imagine applications for very small reactors able to produce discrete, picoliter amounts of product in a highly controllable manner. For example, a system that could be placed on-chip to reform a liquid hydrocarbon to a usable fuel.

Heat is necessary for a variety of heterogeneous catalytic reactions. Many reactions are endothermic, and it is often the case that heat must also be added to create and maintain the vapor phases of liquid reactants and products. Nanoparticles represent an important class of catalysts, and providing heat to them commonly involves transferring heat from a macroscopic source either by conduction via the catalyst's supporting material or by convection through the gas phase, as in the case of automobile exhausts.

Heat transfer to nanoparticle catalysts presents challenges for miniaturization. In microreactors, electrical heaters are common, ${ }^{1,2}$ although novel approaches such as catalytic combustion have also been demonstrated. ${ }^{3}$ When considering

\footnotetext{
* Corresponding author, daboyd@caltech.edu

California Institute of Technology.

* Ecole Polytechnique Fédérale de Lausanne.
}

heat transfer to nanoscale catalysts, it is the total thermal mass of the system, which will be dominated by the catalyst's support material, reactor hardware, etc., that will ultimately determine how small the process can be made and how quickly it can be controlled. For both control of the process and miniaturization, it is clear that one would like to minimize the thermal mass of the system.

In this paper, we demonstrate a technique that we refer to as plasmon-assisted catalysis (PAC). The technique takes advantage of a resonant electronic phenomenon that can occur in metal nanoparticles to directly heat the catalyst particles using a low-power laser. This direct "plasmon heating" of the catalyst particles allows many orders of magnitude reduction in the thermal mass of the reactor system, which in turn allows the process to be miniaturized. We demonstrate PAC by catalytic steam reforming of ethanol inside a microchannel at room temperature.

Nanoparticles have long been used in a wide variety of large scale industrial catalysis. Historically, the push for nanoscale catalysts has been for geometric reasons, i.e., an increase in the number of active sites. However, advances in nanofabrication techniques, mainly the ability to achieve a high degree of particle size uniformity, have enabled researchers to demonstrate that at the nanometer length scale materials can have significantly different catalytic properties than their bulk counterparts due to number of nongeometric effects including changes in the electronic structure, catalyst-substrate interactions, and morphological features. ${ }^{4-6}$ An example is the pioneering work of Haruta in the early 1980s that demonstrated gold, which is considered 
inactive in the bulk, can be highly active when the particle size is only a few nanometers and is supported by titania. ${ }^{7}$

The optical properties of nanoscale particles can also be fundamentally distinct from their bulk counterparts. Noble metal nanoparticles, which serve as catalysts in a number of systems, can strongly couple to visible light. This results from the excitation of surface plasmons (a nearly free electron oscillations coupled to an incident electromagnetic wave) by an external electromagnetic field. Resonant coupling is achieved when the plasmon energy and the particle size satisfy certain shape-dependent resonance conditions. The excitation of plasmon resonances has been used to perform photocatalysis and charge transfer. ${ }^{8,9}$

The "plasmon resonance" is however lossy, and the absorbed energy is quickly converted into heat. In addition, if the particles are supported by a solid, heat transfer from the particles does not necessary obey classical heat conduction. ${ }^{10}$ Taken together, these two effects can allow significant photothermal heating of nanoparticles with surprisingly small amounts of optical energy and without significant heating of the supporting structure.

Plasmon heating allows for the fine control of the location, magnitude, and duration of thermal energy, and this ability to carefully control heating has attracted interest as a means for material deposition and modification..$^{11}$ In contrast, when the nanoparticles are surrounded by a fluid rather than vacuum, the thermal energy can be quickly transferred to the liquid. At very modest laser powers, this allows for optical manipulation of the fluid by rapid evaporation. ${ }^{12,13}$ The ability to directly heat a noble metal nanoparticle in a liquid and produce a vapor phase with an optical wavelength laser suggests that plasmon heating could be applied to heterogeneous catalysis. For liquid reactants, the ability to vaporize the reactants locally is appealing for miniaturization, as it would not require the entire system to be above the boiling point of the reactants to maintain the vapor phase without condensing.

The method of PAC is shown in Figure 1. We will discuss the case for liquid reactants, but the process should work similarly for the gas phase. Catalyst particles are laid down on a substrate by any suitable technique. A liquid is allowed to flow over the catalyst particles. Unlike a typical reactor where the entire system is held for considerable time at the process temperature, which is at least above the boiling point of the reactants, the entire system can be held at room temperature.

To initiate heterogeneous catalysis, a low-power laser with a wavelength coinciding with the plasmon resonance of the catalyst particles is focused onto the substrate. The laser resonantly excites the electronic plasmon modes in the nanostructure, and this energy is converted to lattice vibrations on a picosecond time scale. This results in very rapid heating of the catalyst particle. Due to the small size of the particles, this heat is quickly transferred to the surrounding fluid causing it to be vaporized, while heat loss from the gold to the substrate via conduction is inhibited because of the poor thermal coupling of the gold particle whose size approaches that of the phonon mean free path of the substrate. ${ }^{10}$
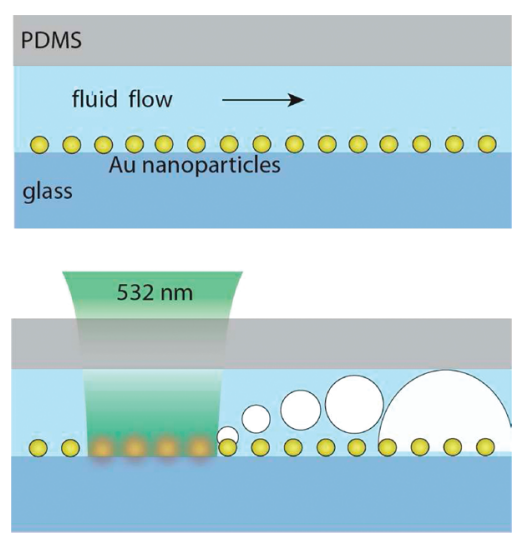

Figure 1. Schematic of the PAC process (side view). A microfluidic channel with gold nanoparticles attached to a glass support; fluid flows from left to right. (Top) A laser at or near the frequency of the plasmon resonance of the gold nanoparticles is focused on the top of the support, and the subsequent heat generated in the nanoparticles is transferred to the surrounding fluid and forms vapor. The vapor phase components react on the catalyst forming gas bubbles which are carried downstream. (The channel height is 40 $\mu \mathrm{m}$ and the radius of the nanoparticles is $\sim 10 \mathrm{~nm}$.)

When the temperature of the plasmon-heated catalyst reaches the range to vaporize the fluid, the heterogeneous catalysis process can begin. The novel aspect of the process is that the heating can be localized in time and space, allowing the use of a high-temperature catalytic process without excessive heating of the immediate surroundings or the preheating needed to vaporize the reactants.

The experiments were conducted within a microfluidic channel, which allowed for precision control of liquid feedstocks and the collection of small amounts of reaction products. A $100 \times 40 \mu \mathrm{m}$ linear microchannel, defined in poly(dimethylsiloxane) (PDMS) (Momentive RTV 615), was temporarily bonded to a glass microscope slide coated with gold nanoparticles. PDMS is transparent, which allows for optical access. The height of the microchannel, $40 \mu \mathrm{m}$, was chosen to avoid heating of the PDMS by the laser. The length of the microchannel was $25 \mathrm{~mm}$, and it had access vias punched at both ends to allow for liquid supply and return. The glass slide was held upside down in the slide holder of a laser microscope (upright configuration). The arrays of gold nanoparticles were produced by block copolymer lithography. ${ }^{14}$ These arrays were deposited on glass microscope slides by spin-coating, as described in our previous work. ${ }^{12}$ The copolymer was polystyrene/poly(vinylpyridine) (PolymerSource P4150-15 VP), with a molecular weight of 55000 AMU PS:50000 AMU PVP. The nanoparticles had an estimated average diameter of $20 \pm 5 \mathrm{~nm}$. A scanning electron microscopy (SEM) image and the optical absorbance spectrum of the particles can be found in the Supporting Information.

The liquid feed for the steam reforming reaction is a 1:1 mixture of doubly distilled water and ACS grade denatured ethanol. The liquid reservoir is a $10 \mathrm{~mL}$ syringe (plunger removed) suspended approximately $0.5 \mathrm{~m}$ above the sample table and connected to the microchannel by a $0.5 \mathrm{~m}$ length of Tygon tubing (0.092 in. i.d.). A $10 \mathrm{~cm}$ length of Tygon tubing is connected to the output port of the channel. Flow 
is established through the microchannel by pulling a partial vacuum on the output tube with a syringe.

A diode-pumped green laser (emission wavelength 532 $\mathrm{nm}$ ) was focused on to the nanoparticle array within the microchannel using a $10 \times$ microscope objective (NA 0.2). The measured beam diameter is $10 \pm 2 \mu \mathrm{m} .{ }^{15}$ The microchannel is observed by a color CCD camera (Sony CCDIRIS) at the microscope's camera port. A laser safety filter is used to cut down on the reflected laser light reaching the camera to prevent saturation of the CCD.

Gas produced in the microchannel was directed into a collection tube for residual gas analysis by quadrupole mass spectroscopy or collected in a syringe for analysis by gas chromatography. The residual gas analyzer (RGA) used was a SRS-RGA 200 (Stanford Research Systems), and it was pumped by a turbomechanical pump backed by a mechanical diaphragm pump (Varian). The turbo pump was connected to the output tube from the microchannel by $3 \mathrm{~m}$ of electroformed nickel tubing (Valco International, 0.0625 in., o.d., 0.040 in., i.d.). A variable leak valve (Duniway Stock Room) was used to set the total RGA chamber pressure. The gas chromatograph (GC) used in this experiment was a HP 5890 II, which has been customized with an array of six external, electronically actuated valves (Valco International). The valve sequence was controlled via LabView and allowed the sample volume to be split between two columns and detectors. The first is a flame ionization detector (FID) with a Mol Sieve 5A column (6 ft. $\times 1 \frac{1}{8}$ in.) for examining fixed gases, e.g., $\mathrm{H}_{2}, \mathrm{O}_{2}, \mathrm{~N}_{2}, \mathrm{CO}_{2}, \mathrm{CO}$, and $\mathrm{CH}_{4}$. The second is a pulsed discharge detector (PDD) with a Hayesep N (6 ft. $\times$ $1 / 8$ in.) column for hydrocarbon separation.

As a control, pure water was first used as the working fluid. At a laser power of $50 \mathrm{~mW}$, we observed a small bubble at the focal point in the microchannel. This bubble was stable and had a diameter of $\sim 10 \mu \mathrm{m}$. If the laser beam was interrupted, the bubble disappeared nearly instantaneously. The bubble would follow the position of the laser spot and could be dragged within the channel by translating the stage. Other than the stationary bubble, we observed no indication of gas or vapor production. We note that the absence of gas production and the ability to drag the bubble in an indiscriminate manner strongly suggest that the vapor bubble is the result of heating rather than a local electronic effect, e.g., a hot spot.

The same procedure was then performed using a 1:1 water to ethanol mixture, and a continuous production of gas was immediately apparent. Unlike the bubble produced in water, these bubbles did not disappear shortly after leaving the area of the laser beam but could be tracked down the length of the channel. An image of the gas plume and the resulting bubble are shown in Figure 2.

The bubbles were generated in a cyclical manner. The evolution of a bubble is shown as a series of images in Figure 3. Initially, a plume of gas emanates from the laser beam spot and produces an expanding bubble, Figure 3a. The bubble grows slowly for several seconds Figure $3 b-d$, and eventually the vapor in the bubble condenses Figure 3e. It then begins to move down the channel, born along by the

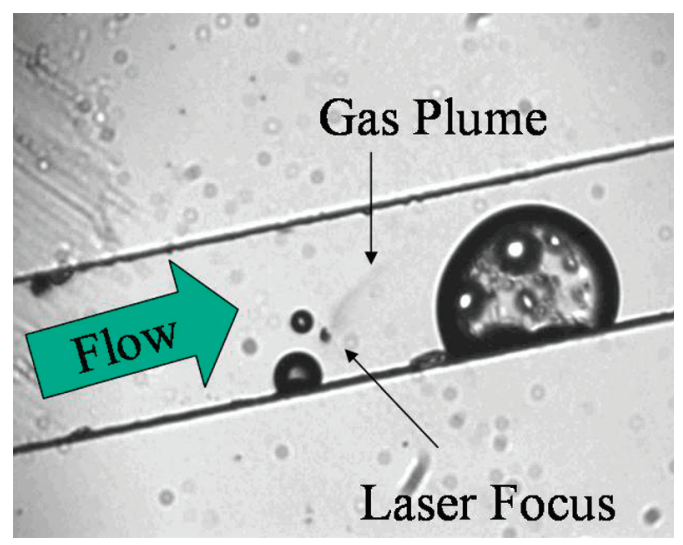

Figure 2. Image of the microchannel taken during PAC (top view). The fluid is 1:1 ethanol and water. A gas plume can be seen emanating from the laser spot and forming bubbles.

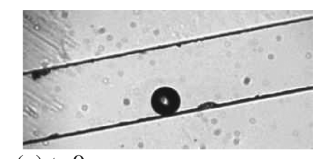

(a) $\mathrm{t}=0 \mathrm{~s}$

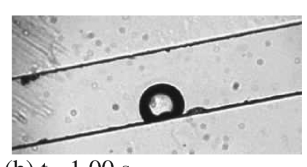

(b) $\mathrm{t}=1.00 \mathrm{~s}$

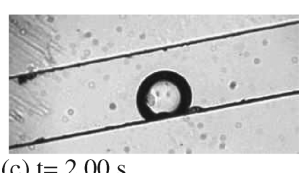

(c) $\mathrm{t}=2.00 \mathrm{~s}$

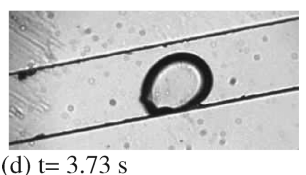

(d) $\mathrm{t}=3.73 \mathrm{~s}$

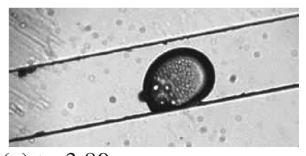

(e) $\mathrm{t}=3.80 \mathrm{~s}$

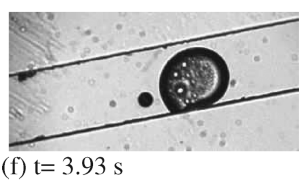

Figure 3. A series of successive images captured during the formation of a single gas bubble (top view). The fluid is a $1: 1$ solution of ethanol and water. A gas bubble is formed $3 \mathrm{a}\left(t=\mathrm{t}_{0}\right)$. The bubble grows in volume $3 \mathrm{~b}(t=1 \mathrm{~s}), 3 \mathrm{c}(t=2 \mathrm{~s}), 3 \mathrm{~d}(t=$ $3 \mathrm{~s})$. Between $3 \mathrm{~d}(t=3.73 \mathrm{~s})$ and $3 \mathrm{e}(t=3.80 \mathrm{~s})$ the vapor in the bubble condenses. Finally, the gas bubble begins to move to the left with the flow $3 \mathrm{f}(t=3.93 \mathrm{~s})$, and a new bubble is nucleated.

flow of liquid. Finally, a new bubble forms, Figure 3f. The period for forming this bubble is about $4 \mathrm{~s}$. As the laser is left on this spot, the rate of bubble production steadily increases. 


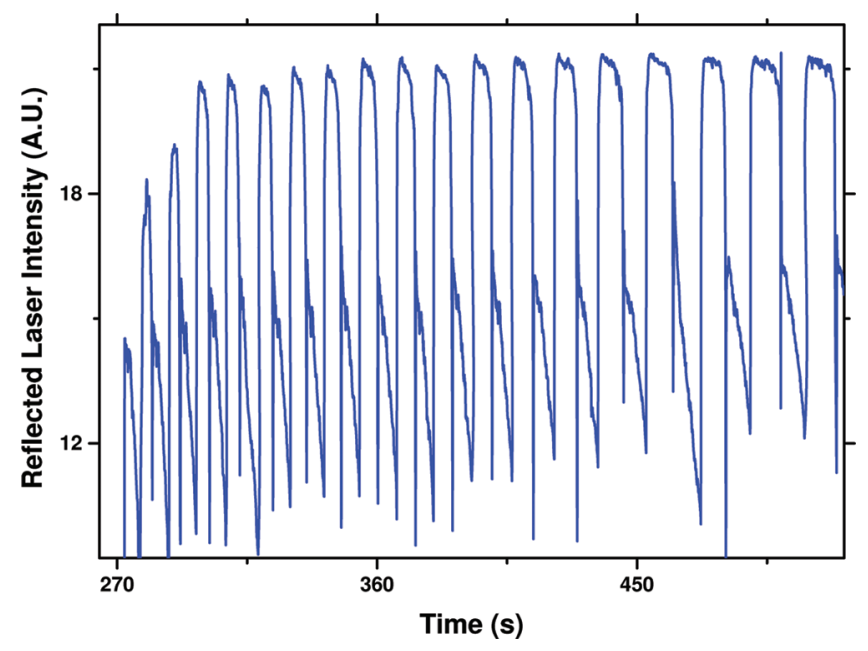

Figure 4. The reflected laser power taken during the PAC process. The oscillations are the result of scatting as gas bubbles are produced within the laser spot. Each cycle corresponds to the formation of a single bubble.

The periodicity of the bubble generation could also be monitored from the reflected laser intensity. Shown in Figure 4 is a plot of the reflected laser power with time as bubbles were generated. The changes in intensity are the result of scattering from the bubble as it forms and then moves out of the beam spot. The period of the cycle was on the order of a few seconds and would gradually increase over a given interval suggesting that larger bubbles were being produced. Monitoring the bubble formation by laser reflection could provide a simple means to monitor the amount of gas generated in future experiments.

After tens of minutes, the bubbling would often become violent, with a plume emanating from the laser focus. The increase in the reaction with time has a memory effect. If we shut off the laser beam for several minutes, and then turn it back on at the same spot, the reaction resumes at a similar rate. If we allow the reaction to run for long periods, darkening of the area and solid deposits become apparent near the laser focus. Examination of the solid deposits with Raman spectroscopy shows that they are consistent with carbon deposition, or so-called coking.

We note that a flow is necessary for PAC to operate continuously. The flow of liquid is estimated to be on the order of $100 \mathrm{~nL} / \mathrm{s}$. Without a flow, we observed that only a single gas bubble would form and grow to a certain size, and it would then remain stationary in the channel. This indicates that the reactions forming the gas had stopped presumably because the reactants had been consumed and no new ones were arriving to the reaction zone.

We used the RGA as described to identify in real time the gas produced by PAC process. The RGA ionizes the gas and records currents at discrete mass to ionization $(\mathrm{m} / \mathrm{z})$ ratios, and the ion currents are proportional to the partial pressure of the particular species. The so-called "cracking pattern" of a substance is the pattern of $\mathrm{m} / \mathrm{z}$ numbers that are observed when that substance is ionized by the RGA. Mass spectra from 1 to $100 \mathrm{~m} / \mathrm{z}$ were taken continuously on the RGA, and the time to take a single spectral scan was

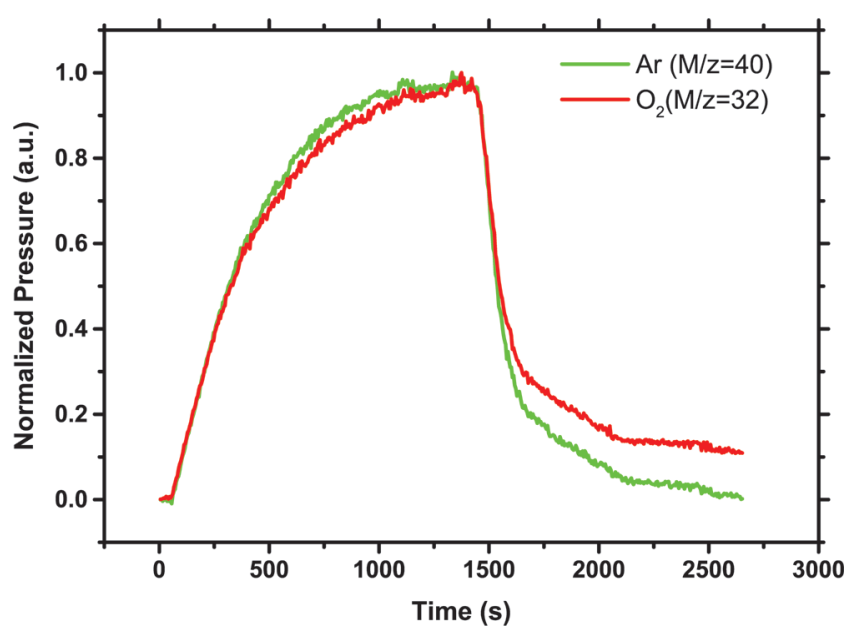

Figure 5. The normalized partial pressures with time of Ar and $\mathrm{O}_{2}$. Time $=0 \mathrm{~s}$ is when the PAC process starts, and it ends at $t=$ $1500 \mathrm{~s}$.

$5 \mathrm{~s}$. The initial background was subtracted from the spectral scans. Cross-correlation analysis was used in part to help determine the products of the reaction. ${ }^{16}$ We used argon as our benchmark gas for the RGA analysis. It is a component of normal atmosphere, present at relatively low concentrations $(1 \%)$, and unambiguously measured by residual gas analysis. Argon shows strong signals at $\mathrm{m} / \mathrm{z}=40$ and $\mathrm{m} / \mathrm{z}$ $=20$, two mass numbers where there is little to no contribution by other molecules appearing in our experiment. We are confident that no chemical or catalytic reaction we could be observing will produce or consume argon. Thus it acts as a good baseline representing the contribution of the atmosphere.

Figure 5 shows the recorded normalized partial pressures both $\mathrm{Ar}$ and $\mathrm{O}_{2}$ during a single run. The PAC process starts at $t=0 \mathrm{~s}$ and is stopped at $1500 \mathrm{~s}$. The pressure rises initially and begins to level-off near $1000 \mathrm{~s}$. This behavior with time is due to the fact that as liquid flows through the microchannel, gas trapped in the sample tube of the RGA is compressed. The increased pressure outside the leak valve increases the amount of gas sampled in any given time step.

Figure 6 shows the recorded partial pressures for $\mathrm{CO}, \mathrm{CO}_{2}$, and $\mathrm{H}_{2}$. Unlike $\mathrm{Ar}$ and $\mathrm{O}_{2}$, the ion currents of $\mathrm{CO}$ and $\mathrm{H}_{2}$ behave in a linear manner with time and do not exhibit the roll-off near $1000 \mathrm{~s}$ as observed with Ar. The behavior of the $\mathrm{CO}_{2}$ is not entirely linear. However, there are substantial amounts of $\mathrm{CO}_{2}$ in the atmosphere, and we expect the partial pressure to be a convolution of the compression of atmospheric gas and the production of gas from the reactions. Such behavior is apparent in Figure 6 as indicated by the increasing pressure values after $1000 \mathrm{~s}$. The similarity in shape of the $\mathrm{CO}, \mathrm{CO}_{2}$, and $\mathrm{H}_{2}$ curves, and the marked difference in shape compared to those of $\mathrm{O}_{2}$ and $\mathrm{Ar}$ are evidence that bubbles produced contain all three of these gases. In addition, the measurements indicate that $\mathrm{O}_{2}$ is not being produced in detectable amounts during the process ruling out electrolysis in this system.

We used the GC to confirm and quantify the reaction products under similar process conditions in separate runs. 


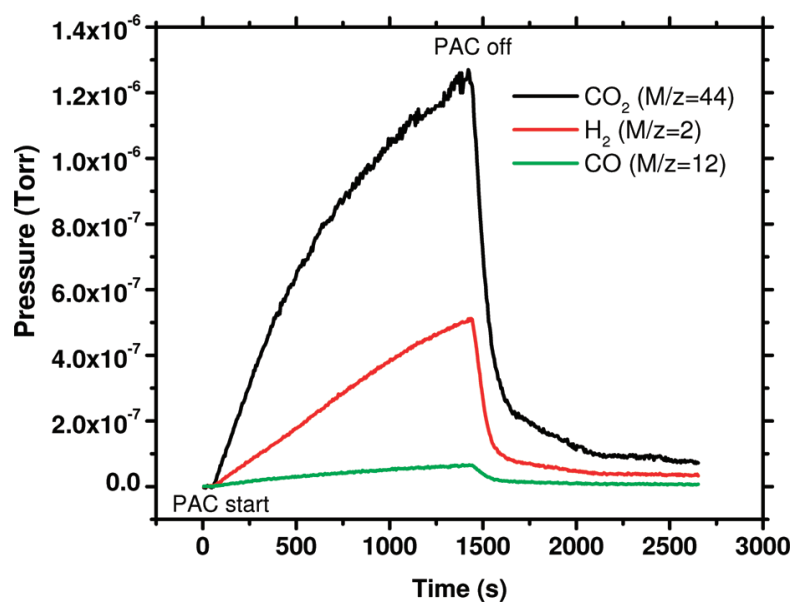

Figure 6. The partial pressures with time of $\mathrm{CO}_{2}(\mathrm{~m} / \mathrm{z}=44), \mathrm{CO}$ $(m / z=12)$, and $\mathrm{H}_{2}(m / z=2)$ as measured by RGA. Time $=0 \mathrm{~s}$ is when the PAC process starts, and it ends at $t=1500 \mathrm{~s}$.

These measurements are complementary to those of the RGA; they provide a definitive, quantitative measurement integrated over the entire run and the ability to unquestionably determine the amount of $\mathrm{CO}$ generated. The PAC process was run with the same 1:1 water to ethanol mixture for $1.5 \mathrm{~h}$, and the gas was collected into a syringe. The volume of the sample was less than $0.1 \mathrm{~mL}$, and to achieve a suitable volume of gas for the GC, the sample was mixed with room air to make a total volume of $3 \mathrm{~mL}$. The gas samples were referenced to the same volume of room air, and gas standards were used to determine the absolute concentrations. The sample loop $(1 \mathrm{~mL})$ was evacuated to a few mTorr before the sample was added. The composition of a typical run was $\mathrm{CO} 14 \mathrm{ppm}, \mathrm{H}_{2} 44 \mathrm{ppm}$, and $\mathrm{CO}_{2} 170$ $\mathrm{ppm}$. The GC confirmed the presence of $\mathrm{CO}$ in the products, and hydrocarbon products were not observed.

The heterogeneous nucleation of a small stationary bubble on the surface of a water-filled channel allows us to estimate the heating of the liquid. As mentioned above, we have observed that a static bubble can be formed by focusing the laser beam under $10 \times$ magnification at $50 \mathrm{~mW}$ in water. We hypothesize that this is a water vapor bubble nucleated at the site of laser heating. The bubble is on the order of 10 $\mu \mathrm{m}$ in diameter. The pressure inside this bubble, the Laplace pressure, is calculated from

$$
\Delta P=2 \gamma / r
$$

where $\gamma$ is the surface energy of water, $\Delta P$ is the pressure difference between the vapor in the bubble and the surrounding fluid, and the $r$ is the radius of the bubble.

Because of the very high pressure of a small bubble such as this one, the liquid must be superheated in order to have enough energy to form and maintain the vapor phase. The temperature $\Delta T$ above the normal boiling point is given by ${ }^{17}$

$$
\Delta T=\frac{R T_{\mathrm{ext}}^{2} 2 \gamma}{l_{\mathrm{H}_{2} \mathrm{O}} P_{\mathrm{liq}} r}
$$

Here $R$ is the gas constant from the ideal gas law, $T_{\text {ext }}$ is the normal boiling point, $P_{\text {liq }}$ is the liquid pressure outside the bubble, and $l_{\mathrm{H}_{2} \mathrm{O}}$ is the latent heat of vaporization for water.

Using the numerical values $\gamma=7.2 \times 10^{-2} \mathrm{~N} / \mathrm{m}, T_{\text {ext }}=$ $373 \mathrm{~K}, R=8.31 \mathrm{~J} \cdot \mathrm{K}^{-1} \cdot \mathrm{mol}, l_{\mathrm{H}_{2} \mathrm{O}}=40680 \mathrm{~J} / \mathrm{mol}$, and $P_{\text {liq }}=$ $101 \mathrm{kPa}$, eq 1 gives a pressure difference of $28 \mathrm{kPa}$ for a bubble of $r=5 \mu \mathrm{m}$, and the superheated temperature of the bubble $\Delta T$ calculated from eq 2 is $108 \mathrm{~K}$. This high temperature and pressure of the vaporized liquid near the laser focus is similar to conditions achieved in catalytic reactors often used to perform steam reforming. Therefore, it is not surprising that we see evidence of the catalytic production of gas bubbles in the case of ethanol-water mixtures.

We now consider laser heating of a nanoparticle in water by a continuous wave laser. The temperature is ultimately determined by the incident power density and the heat transfer from the nanoparticles to the surrounding fluid. We neglect heat transfer to the substrate as this will be reduced due to the size of the nanoparticles relative to the phonon mean free path of the glass substrate. ${ }^{10}$ Following the approach of Pustovalov, ${ }^{18}$ the temperature of a spherical particle due to a power density $I_{0}$ in the steady state can be shown to be

$$
T_{0}=T_{\infty}+\frac{I_{0} K_{\mathrm{abs}} r_{0}}{4 k_{\infty}}
$$

where $K_{\text {abs }}$ is the efficiency absorption factor for a particle of radius $r_{0}$, which can be calculated from Mie scattering theory, ${ }^{19}$ and $k_{\infty}$ is the coefficient of thermal conductivity of the surrounding medium at the macroscopic equilibrium temperature $T_{\infty}$. To calculate the temperature rise in a nanoparticle of $25 \mathrm{~nm}$ in diameter, we use the following values: the ambient temperature $T_{\infty}$ is $25^{\circ} \mathrm{C}$; the value of $k_{\infty}$ is $0.6 \mathrm{~W} / \mathrm{m} \cdot \mathrm{K}$, which is the known value for water; the power density $I_{0}$ is $6.4 \times 10^{8} \mathrm{~W} / \mathrm{m}^{2}$, and we set $K_{\text {abs }}=2.0$. These give a value of $T_{0}=34{ }^{\circ} \mathrm{C}$. We note these numbers in our calculations are all approximate and affected by a number of additional factors but are presented here only to illustrate that temperature rises of the order required to explain our results are quite likely.

On the basis of the nanparticle heating, our result is surprising since we are clearly able to generate a superheated water vapor bubble under these conditions. A possible explanation for this behavior is that we are able to nucleate a bubble from dissolved or absorbed gas or from an instability around the metal nanoparticles caused by the intense electric fields generated by the plasmon resonance. The thermal conductivity of water vapor is $0.015 \mathrm{~W} / \mathrm{m} \cdot \mathrm{K}$, and a nucleated bubble would dramatically reduce the heat transfer from the nanoparticles to the fluid. This along with reduced heat transfer from the nanoparticles to the substrate, which is believed to occur as a result of nonclassical effects, ${ }^{10,11}$ would allow the particle to reach and sustain much higher temperatures than otherwise possible. The origin of this type of bubble 
nucleation is beyond the scope of this work but is a subject for future studies.

The overall reaction for the reforming of ethanol to hydrogen can be described $\mathrm{as}^{20}$

$$
\mathrm{C}_{2} \mathrm{H}_{5} \mathrm{OH}+3 \mathrm{H}_{2} \mathrm{O} \rightarrow 2 \mathrm{CO}_{2}+6 \mathrm{H}_{2}
$$

Depending on the catalyst and the conditions, there are a number of pathways, including decomposition of ethanol followed by steam reforming ${ }^{21}$ and direct steam reforming of ethanol to syngas. ${ }^{22}$ In addition to forming $\mathrm{CO}_{2}$ and $\mathrm{H}_{2}$, $\mathrm{CO}$ is a biproduct in these reactions, but it can react with steam over the catalyst to produce $\mathrm{CO}_{2}$ and $\mathrm{H}_{2}$. This is known as the water-gas shift reaction

$$
\mathrm{CO}+\mathrm{H}_{2} \mathrm{O} \rightarrow \mathrm{CO}_{2}+2 \mathrm{H}_{2}
$$

These equations represent model reactions involving an ideal catalyst. However, depending on the reaction conditions, e.g., the catalyst temperature, there are a number of possible reaction pathways that can occur making the overall reaction quite complex. For example, in our studies we measure an excess of $\mathrm{CO}_{2}$ and the presence of $\mathrm{CO}$. This suggests that the conditions are not optimal for the water-gas shift reaction. Haryanto et al. provide a current review of steam reforming of ethanol. ${ }^{23}$

The efficiency of the reforming will depend on the catalyst that is used. Common materials and material systems for catalytic steam reforming include oxide catalysts and oxidesupported metal catalysts. For example, in the oxidesupported metal catalyst system $\mathrm{Co}$ on $\mathrm{Al}_{2} \mathrm{O}_{3}$, it is reported that this system can convert $100 \%$ of the ethanol with selectivities of $67 \%, 19 \%, 7.9 \%$, and $6.0 \%$ for $\mathrm{H}_{2}, \mathrm{CO}_{2}, \mathrm{CO}$, and $\mathrm{CH}_{4}$, respectively. ${ }^{24}$ From our GC measurements, we observe selectivities of $19 \%, 75 \%$, and $6 \%$ for $\mathrm{H}_{2}, \mathrm{CO}_{2}$, and $\mathrm{CO}$, respectively. We did not observe the presence of $\mathrm{CH}_{4}$. Coking of the sample could be the result of decomposition of $\mathrm{CH}_{4}$, polymerization of $\mathrm{C}_{2} \mathrm{H}_{4}$, or from the Bourdouard reaction, ${ }^{23}$ and it may in part be attributed to the occasional air bubble that passed within the reaction zone, which caused the liquid in the area to dry out. The catalytic disproportionation of $\mathrm{CO}$ to carbon clusters has also been observed on roughened gold films. ${ }^{25,26}$ It is not clear from the current studies exactly how the presence of carbon effects the catalytic activity of the gold nanoparticles, but it does not seem to inhibit the gas generation process.

High selectivities values for hydrogen are desirable. In our system we consider both the catalyst system and the temperature of the catalyst to be factors for the low observed value of hydrogen. In general, bulk gold is not considered to be an active metal catalyst. However, gold nanoparticles on a support such as titania or ceria can have good activity, and these systems have been used for performing the water-gas shift (WGS) reaction at low temperatures. ${ }^{27}$ The temperature of the catalyst can also affect the activity. For the given laser power density used in experiment, the local temperatures achieved, at least $\sim 408 \mathrm{~K}$, may not have been high enough for optimal performance. For example, rhodium mixed with ceria supported by alumina is considered to be an excellent system for authothermal reforming of ethanol and works optimally at or above $973 \mathrm{~K}^{22}$

In this current work, we did not explore the mass-energy balance of the ethanol conversion. The width of our microfluidic channels was much larger than the beam waist so that much of the flowing reactants did not enter the reaction zone. The total flow of reactants was not able to be measured with high precision; we used pressure driven pumping, and the flow was not necessarily constant making it difficult to accurately account for exactly how much fluid entered into the reaction zone per given unit of time. Future studies of this system would involve regulated flows of the reactants as well as channels widths closer to the beam diameter. In addition, a comparison to gold nanoparticles on glass heated by conventional means, e.g., an oven, would be useful to understand the catalytic role of gold in the process.

In conclusion, we have demonstrated a method capable of miniaturizing and performing many types of heterogeneous catalysis. Our approach is to optically heat nanoparticle catalysts directly via plasmon resonance using low-power lasers. An advantage of this method is that the process can be highly localized and avoids the need for external wiring, heated reactant lines, and or maintaining the entire system at or above the reaction temperature. This in turn allows the process to be incorporated within proximity to environments that may not be able to withstand high temperatures, e.g., electronic circuits. The recent development of highly integrated microfluidic systems for optofluidics may provide a framework for the eventual incorporation of PAC into of efficient microcatalysis units for fuel conversion or chemical synthesis in lab-on-a-chip applications.

Clearly PAC may not be desirable for producing large volumes of product without significantly more laser power. However, as demonstrated it does provide a compact and highly controllable means for studying catalytic, especially photocatalytic, reactions under conditions approaching that of a continuous flow reactors. This approach could prove complementary to studies of well-characterized single crystals in ultrahigh vacuum, which have contributed to the understanding of the basic reaction pathways. In addition, we note that that ability to monitor the regular formation of bubbles could allow for a simple approach to monitoring gas production.

A challenge faced by PAC is that not all catalysts possess a plasmon resonance. However, there are number that do including $\mathrm{Rh}$ and $\mathrm{Cu}$, and as well as Au nanoparticles on an active supports such as $\mathrm{TiO}_{2}$ or $\mathrm{CeO}_{2}$. These systems, along with studies of the efficiency of PAC, are subjects for future studies.

Acknowledgment. This work has been generously supported by the DARPA Center for Optofluidic Integration by Award Number HR0011-04-1-0032 and the Global Climate and Energy Program (GCEP), Stanford University.

Supporting Information Available: SEM image of gold nanoparticles and optical absorbance of gold nanoparticles. 
This material is available free of charge via the Internet at http://pubs.acs.org.

\section{References}

(1) Pattekar, A.; Kothare, M. J. Microelectromech. Syst. 2004, 13, 7-18.

(2) Men, Y.; Kolb, G.; Zapf, R.; Hessel, V.; Löwe, H. Process Saf. Environ. Prot. 2007, 85, 413-418.

(3) Park, D.-E.; Kimb, T.; Kwon, S.; Kima, C.-K.; Yoon, E. Sens. Actuators, A 2007, 135, 58-66.

(4) Cho, A. Science 2003, 299, 1684-1685.

(5) Meyer, R.; Lemire, C.; Shaikhutdinov, S. K.; Freund, H.-J. Gold Bull. (London, U.K.) 2004, 37, 72-124.

(6) Haruta, M. CATTECH 2002, 6, 102-115.

(7) Haruta, M.; Kobayashi, T.; Sano, H.; Yamada, N. Chem. Lett. 1987, 2, 405-408.

(8) Awazu, K.; Fujimaki, M.; Rockstuhl, C.; Tominaga, J.; Murakami, H.; Ohki, Y.; Yoshida, N.; Watanabe, T. J. Am. Chem. Soc. 2008, 130, 1676-1680.

(9) Tian, Y.; Tatsuma, T. J. Am. Chem. Soc. 2005, 127, 7632-7637.

(10) Chen, G. J. Heat Transfer 1996, 118, 539-545.

(11) Boyd, D. A.; Greengard, L.; Brongersma, M.; El-Naggar, M.; Goodwin, D. Nano Lett. 2006, 6, 2592-2597.

(12) Boyd, D. A.; Adelman, J.; Goodwin, D. G.; Psaltis, D. Anal. Chem. 2008, 80, 2452-2456.
(13) Liu, G. L.; Kim, J.; Lu, Y.; Lee, L. P. Nat. Mater. 2006, 5, 27-32.

(14) Spatz, J. P.; Mossmer, S.; Hartmann, C.; Möller, M. Langmuir 2000, 407, 407-415.

(15) Cohen, D. K.; Little, B.; Luecke, F. S. Appl. Opt. 1984, 3, 637-640.

(16) Noda, I. Appl. Spectrosc. 2000, 54, 994-999.

(17) Thome, J. Heat Transfer Handbook; Wiley-IEEE: Hoboken, NJ, 2003; pp 640-644.

(18) Pustovalov, V. K. Chem. Phys. 2005, 308, 103-108.

(19) Pustovalov, V. K.; Babenko, V. A. Laser Phys. Lett. 2004, 1, 516520.

(20) Velu, S.; Satoh, N.; Gopinath, C. S.; Suzuki, K. Catal. Lett. 2002, 82, $145-152$.

(21) Fatsikostas, A. N.; Verykios, X. E. J. Catal. 2004, 225, 439-452.

(22) Deluga, G. A.; Salge, J. R.; Schmidt, L. D.; Verykios, X. E. Science 2004, 303, 993-997.

(23) Haryanto, A.; Fernando, S.; Murali, N.; Adhikari, S. Energy Fuels 2005, 19, 2098-2106.

(24) Haga, F.; Nakajima, T.; Miya, H.; Mishima, S. Catal. Lett. 1997, 48, 223-227.

(25) Kudelski, A.; Pettinger, B. Chem. Phys. Lett. 2004, 383, 76-79.

(26) Kudelski, A.; Pettinger, B. Chem. Phys. Lett. 2000, 321, 356-362.

(27) Andreeva, D.; Idakiev, V.; Tabakova, T.; Ilieva, L.; Falaras, P.; Bourlinos, A.; Travlos, A. Catal. Today 2002, 72, 51-57.

NL902711N 